Memoirs of The College of Science, University of Kyoto, Series A Vol. XXVIII, Mathematics No. 3, 1953.

\title{
Stationary random distributions
}

By

Kiyosi ITô

(Received April 15, 1954)

In the same way as the concept of distributions by L. Schwartz $[11]^{1)}$ was introduced as an extended one of functions, we may define stationary random distributions as an extension of stationary random functions viz. stationary processes. Such consideration will enable us to establish a unified theory of stationary processes, Brownian motion processes, processes with stationary increments and other similar ones, as is shown in this paper. We shall first introduce some fundamental notions in $\S 1$. In $\S 2$ we shall define the covariance distribution of stationary random distributions, which corresponds to Khintchine's covariance function [7]. In $\S 3$ and $\S 4$ we shall prove the spectral decomposition theorems of covariance distributions and stationary random distributions respectively. In $\S 5$ we shall discuss the derivatives of stationary distributions. In $\S 6$ we shall show that any stationary distribution is identified with a $k$-th derivative (in the sense of distributions) of a certain continuous process with stationary $k$-th order increments for some $k$.

\section{§1. Fundamental Notions and Notations}

In this paper we shall restrict ourselves to complex-valued random variables with mean 0 and finite variance. The totality of such variables constitute a Hilbert space $\mathfrak{S}$ with the following definition of inner product:

$$
(X, Y)=\bar{\sigma}(X \cdot \bar{Y}) ; \quad \bar{\sigma}: \text { expectation. }
$$

We shall here consider only the strong topology on $\mathfrak{g}$. A continuous random process $X(t),-\infty<t<\infty$, is an $\mathfrak{S}$-valued continuous

1) The numbers in [ ] refer to those of the Bibliography at the end of this paper. 
function defined on $R=(-\infty, \infty)$. The set of all continuous processes will be denoted by $G(\mathfrak{T})$.

Let $D$ denote the space of all complex-valued $C_{\infty}$-functions defined on $R$ whose carrier is compact. We shall introduce in $\nexists$ the topology of Schwartz [11] (III, $\S 1$ ). An $\mathfrak{S}$-valued continuous linear functional difined on $D$ is called a random distribution. We shall here denote with $D^{\prime}(\mathfrak{S})$ the totality of random distributions, while $D^{\prime}$ will denote the set of all complex-valued distributions (continuous linear functionals) defined on $D$. $b(\mathfrak{S})$ may be considered as a sub-system of $D^{\prime}(\mathfrak{g})$, since we may identify a continuous random process $X(t)$ with the following random distribution $X(\phi)$ determined by it :

$$
X(\phi)=\int X(t) \phi(t) d t ;
$$

the integral sign without bounds means the integral from $-\infty$ to $\infty$ in this paper.

The following notations will be often used in the theory of distributions. Let $F \in D^{\prime}$ or $D^{\prime}(\mathfrak{g})$ and $\phi \in D$.

$\tau_{h}$ (shift transformation) : $\tau_{h} \phi(t)=\phi(t+h), \tau_{h} F(\phi)=F\left(\tau_{-h} \phi\right)$

$D$ (Derivative) : $D \phi(t)=\phi^{\prime}(t), D F(\phi)=-F(D \phi)$

$\sim$ (Inversion) : $\check{\phi}(t)=\phi(-t), \check{F}(\phi)=F(\check{\phi})$

- (Conjugate) : $\bar{\phi}(t)=\overline{\phi(t)}, \bar{F}(\phi)=\overline{F(\bar{\phi})}$

$\sim(=\bar{v}=\simeq) \quad: \tilde{\phi}(t)=\overline{\phi(-t)}, \tilde{F}(\phi)=\overline{F(\tilde{\phi})}$

$\mathscr{f}$ (Fourier transform) : $\mathscr{f} \phi(\lambda)=\int e^{-i 2 \pi \lambda t} \phi(t) d t$

The following relations should be noted.

$$
(F * \phi)(0)=F(\check{\phi})=\check{F}(\phi), \quad \mathcal{F}(\phi * \psi)=\not{\not} \phi \cdot \not{\not} \phi, \quad \mathscr{f} \tilde{\phi}=\bar{\not} \phi
$$

We shall call, following Khintchine, $X \in \ell(\mathfrak{g})$ weakly stationary or merely stationary for the brevity if its covariance function is invariant under shift transformations viz. if we have, for any $(t, s)$,

$$
\left(\tau_{h} X(t), \tau_{h} X(s)\right)=(X(t), X(s)),
$$

and to be strictly stationary if its probability law is invariant under shift transformations viz. if the joint probability law of

$$
\left(\tau_{h} X\left(t_{1}\right), \cdots, \tau_{h} X\left(t_{n}\right)\right)
$$

is independent of $h$ for any $n$ and any $\left(t_{1}, \cdots, t_{n}\right)$. Generalizing these definitions onto random distributions, we shall call $X \in D^{\prime}(\mathfrak{C})$ weakly 
stationary or merely stationary for the brevity if we have, for any $\left(\phi, \phi^{\prime}\right)$,

$\left(1 \cdot 4^{\prime}\right) \quad\left(\tau_{h} X(\phi), \tau_{h} X\left(\phi^{\prime}\right)\right)=\left(X(\phi), X\left(\phi^{\prime}\right)\right)$

and strictly stationary if the joint probability law of

$$
\left(\tau_{h} X\left(\phi_{1}\right), \cdots, \tau_{h} X\left(\phi_{h}\right)\right)
$$

is independent of $h$ for any $n$ and $\left(\phi_{1}, \cdots, \phi_{n}\right)$.

We shall adopt here the following notations:

$\mathfrak{S}:$ the totality of stationary distributions,

$\mathfrak{S}^{\circ}$ : the totality of stationary processes,

$\overline{\mathfrak{S}}$ : the totality of strictly stationary distributions,

$\bar{\complement}^{\circ}$ : the totality of strictly stationary processes.

Clearly we have

$$
\mathfrak{S} \supseteq \overline{\mathfrak{S}} \mathfrak{S}^{\circ}, \quad \mathfrak{S}^{\circ} \supseteq \overline{\mathfrak{S}}^{\circ} \text {. }
$$

A random distribution $X$ is called a complex normal (random) distribution if $X(\phi), \phi \in D$, constitute a complex normal system [5], and to be a real normal (random) distribution if $X$ is real viz. $X=\bar{X}$ and $X(\phi), \phi$ running over real functions in $D$, constitute a (real) normal system. This is an extention of normal processes or Gaussian processes [3] $(\mathrm{II}, \S 3)^{2)}$. $A$ (complex as well as real) normal distribution will be strictly stationary, if it is weakly stationary. The corresponding fact is well-known regarding stationary processes.

We shall here mention a typical example of real stationary distributions which are not stationary processes. Let $B(t)$ be a (real) Brownian motion process [3] (p. 97). The derivative (in the sense of distribution) of this process $B^{\prime} \equiv D B$ is a random distribution defined by

$$
\text { (1.7) } \quad B^{\prime}(\phi)=-B\left(\phi^{\prime}\right)=\int \phi(t) d B(t) \quad \text { (Wiener integral) [4]. }
$$

This is evidently real normal and stationary, since

$$
\begin{aligned}
& \left(\tau_{h} B^{\prime}(\phi), \tau_{h} B^{\prime}(\phi)\right)=\left(B^{\prime}\left(\tau_{-h} \phi\right), B^{\prime}\left(\tau_{-h} \phi\right)\right) \\
& =\left(\int \phi(t-h) d B(t), \int \phi(t-h) d B(t)\right) \\
& =\int \phi(t-h) \overline{\phi(t-h)} d t=\int \phi(t) \overline{\zeta^{\prime \prime}(t)} d t,
\end{aligned}
$$

2) This definition of complex normal ones is somewhat different from that of Doob, while both definitions are the same in the real case. 
which shows that $B^{\prime} \in \mathfrak{S}$. The fact that $B^{\prime} \bar{\epsilon} \mathfrak{S}^{\circ}$ will be proved in $\S 2$.

\section{§2. Covariance Distribution}

As is known, Khintchine's covariance function [7] $\rho(t)$ of $X(t)$ is defined by

$$
\rho(t)=(X(t), X(0))=(X(t+s), X(s)) .
$$

Then we have clearly

$$
\begin{aligned}
\left(X(\phi), X\left(\psi^{\prime}\right)\right) & =\left(\int X(t) \phi(t) d t, \int X(s) \psi^{\prime}(s) d s\right) \\
& =\iint(X(t), X(s)) \phi(t) \overline{\psi^{\prime}(s)} d t d s \\
& =\iint \rho(t-s) \phi(t) \overline{\psi(s)} d t d s \\
& =\int \rho(\tau) \int \phi(\tau-\sigma) \overline{\psi(-\sigma)} d \sigma d \tau .
\end{aligned}
$$

By considering $\rho$ as a distribution $\epsilon D^{\prime}$, we may write the above identity as

$$
\left(X(\phi), X\left(\phi^{\prime}\right)\right)=o\left(\phi * \phi^{\prime}\right) .
$$

This may suggest to us how to define the covariance distribution of our stationary distributions.

THEOREM $2 \cdot 1$. Let $X(\phi)$ be any stationary distribution. Then there exists one and only one distribution $\rho \in D^{\prime}$ satisfying $(2 \cdot 2)$.

DEFINITION. This distribution is called the covariance distribution of $X$.

PROOF of THEOREM. If we put

$$
T_{\phi}(\phi)=(X(\phi), X(\bar{\phi})),
$$

then we get a distribution $T_{\phi} \in D^{\prime}$ for each $\phi \in D$. Taking into account the fact that $T_{\phi}(\psi)$ is continuous in $(\phi, \psi) \in D \times D$ and $D$ is a Montel space [11] (III, $\S 2$ ), we shall easily see that ' $\phi \rightarrow T_{\phi}$ ' is a continuous linear mapping from $D$ into $D^{\prime}{ }^{3}$.) Furthermore this transformation commutes with the shift transformation;

$$
\begin{aligned}
\left(\tau_{h} T_{\phi}\right)\left(\phi^{\prime}\right) & \left.=T_{\phi}\left(\tau_{-h} \phi^{\prime}\right)=\left(X(\phi), X \overline{\left(\tau_{-h} \phi^{\prime}\right.}\right)\right)=\left(X(\phi), X\left(\tau_{-h} \bar{\phi}\right)\right) \\
& =\left(X\left(\tau_{h} \phi\right), X(\bar{\psi})\right)=T_{\tau_{h} \phi^{\phi}}\left(\phi^{\prime}\right) .
\end{aligned}
$$

Therefore $T_{\phi}$ is expressible as a convolution of a distribution $T$ and $\phi$ :

3) Cf. [11] III, $\S 3$ as to the topology in $\bigotimes^{\prime}$. 


$$
T_{\phi}=T * \phi,
$$

by a theorem of Schwartz [11] (Vol. 2, VI, $\S 7$, p. .53, Amélioration $\mathrm{du}$ théorèm $\mathrm{X})$. Hence it follows that

$$
(X(\phi), X(\phi))=T_{\phi}(\bar{\psi})=(T * \phi)(\bar{\psi})=(T * \phi * \tilde{\psi})(0)=\rho(\phi * \tilde{\psi}),
$$

where

$$
\rho=\check{T} \text {. }
$$

The uniqueness of $\rho$ follows at once from the fact that the set of all elements of the form $\phi * \phi, \phi, \psi \in D$, is dense in $D$.

THEOREM 2.2. If $X(\phi)$ is real stationary distribution, then its covariance distribution is real, i.e. $\rho=\bar{\rho}$.

PROOF. Let $\rho$ be the covariance distribution of $X$. Then that of $\bar{X}$ will become $\bar{\rho}$, since we have

$$
\begin{aligned}
(\bar{X}(\phi), \bar{X}(\psi)) & =(\overline{X(\bar{\phi})}, \overline{X(\bar{\psi})})=\overline{(X(\bar{\phi}), X(\bar{\psi})})=\overline{\rho(\bar{\phi} * \tilde{\bar{\psi}})} \\
& =\overline{\rho(\phi * \tilde{\psi})}=\bar{\rho}(\phi * \tilde{\psi}) .
\end{aligned}
$$

Thus $X=\bar{X}$ implies $\rho=\bar{\rho}$.

EXAMPLE. The covariance distribution of $B^{\prime}$ is Dirac's $\delta$-distribution [11] (I, §1), as

$$
\begin{aligned}
\left(B^{\prime}(\phi), B^{\prime}(\phi)\right) & =\int_{\phi} \phi(t) \overline{\psi(t)} d t=\int \phi(t) \tilde{\psi}(-t) d t=(\phi * \tilde{\psi})(0) \\
& =\delta(\phi * \phi) .
\end{aligned}
$$

Thus we see that $B^{\prime} \bar{\epsilon} \mathfrak{S}^{\circ}$, because if $B^{\prime} \in \mathfrak{S}^{\circ}$ then the covariance distribution would be induced by a continuous function which is Khintchine's covariance function.

\section{§3. Spectral Decomposition of Covariance Function}

Let $X(t)$ be any stationary continuous random process with covariance function $\rho(t)$. A known theorem of Khintchine [7] shows that $\rho(t)$ is expressible in the form:

$$
\rho(t)=\int e^{-i 2 \pi \lambda \ell} d \mu(\lambda)
$$

in one and only one way, where $\mu$ is a non-negative measure on $R$ such that $\mu(R)<\infty$. Therefore the distribution $\rho(\phi)$ induced by $\rho(t)$ may be expressed as

$$
\rho(\phi) \equiv \int \imath(t) \phi(t) d t=\int \not{f} \phi(\lambda) d \mu(\lambda) .
$$

Let us now consider any stationary distribution with the covariance distribution $\rho$. Then we have 


$$
\rho(\phi * \widetilde{\phi})=(X(\phi), X(\phi)) \geqq 0,
$$

which implies that $\rho$ is a positive-definite distribution. By Schwartz' generalization of Bochner's theorem [11] (VII, $\$ 9$ ) we obtain the following theorem.

THEOREM 3.1. $\rho$ is expressible in the from $(3 \cdot 2)$ in one and only way, where $\mu$ is a non-negative measure satisfying

$$
\int \frac{d \mu(\lambda)}{\left(1+i^{2}\right)^{k}}<+\infty
$$

for some integer $k$.

DEFINITION. We shall call $(3 \cdot 2)$ the spectral decomposition of $\rho$ and $\mu$ spectral measure of $\rho$. When $\mu$ satisfies (3.4); we say that $X \in \mathfrak{S}_{k}$. We have clearly

$$
\cdots \mathfrak{S}_{-2} \subseteq \mathfrak{S}_{-1} \subseteq \mathfrak{S}_{0} \subseteq \mathfrak{S}_{1} \subseteq \mathfrak{S}_{2} \subseteq \cdots .
$$

Conversely we have

ThEOREM 3.2. Any distribution of the above form is the covariance distribution of a stationary distribution which is complex normal.

Proof. Let $\rho$ be a distribution of the above form. Put

$$
\Gamma(\phi, \psi)=\rho(\phi * \tilde{\psi}), \phi, \phi \in \mathcal{D} .
$$

Then $\Gamma(\phi, \psi)$ is positive-definite in $(\phi, \phi)$, as we have

$$
\sum_{i, j=1}^{n} \Gamma\left(\phi_{i}, \phi_{j}\right) \xi_{i} \bar{\xi}_{j}=\rho(\theta * \tilde{\theta}) \geqq 0, \quad \theta=\sum_{i} \xi_{i} \phi_{i} .
$$

Therefore we can define a complex normal system $X(\phi), \phi \in \mathcal{D}$, such that $\bar{\sigma} X(\phi)=0$ and $\left.\bar{\sigma}\left(X(\phi) \cdot \overline{X\left(\psi^{\prime}\right.}\right)\right)=\Gamma(\phi, \phi)=\rho(\phi * \widetilde{\phi})[5]$. It remains only to show that $X(\phi)$ is a random distribution. From the identity :

$$
\begin{aligned}
\|X(c \phi)-c X(\phi)\|^{2}= & (X(c \phi), X(c \phi))-c(X(\phi), X(c \phi)) \\
& --\bar{c}(X(c \phi), X(\phi))+c \bar{c}(X(\phi), X(\phi)) \\
= & \prime(c \phi * \widetilde{c \phi})-c \rho(\phi * \widetilde{c \phi})-\bar{c} \rho(c \phi * \widetilde{\phi})+c \bar{c} \rho(\phi * \widetilde{\phi}) \\
= & c \bar{c} \rho(\phi * \widetilde{\phi})-c \bar{c} \rho(\phi * \widetilde{\phi})-c c \rho(\phi * \tilde{\phi})+c \bar{c} \rho(\phi * \tilde{\phi}) \\
= & 0,
\end{aligned}
$$

it follows that $X(c \phi)=c X(\phi)$. By the same way we can see that $X(\phi+\psi)=X(\phi)+X(\phi)$. Therefore $X(\phi)$ is linear in $\phi$. By the 
identity $\|X(\phi)\|^{2}=\rho(\phi * \widetilde{\phi})$ we obtain the continuity of $X$. Thus our theorem is completely proved.

Next we shall discuss the case of real stationary distributions. By Theorem $2 \cdot 2$ we see that $\rho=\bar{l}$ in this case. But we have

$$
\begin{aligned}
\bar{\rho}(\phi) & =\overline{\rho(\bar{\phi})}=\int \overline{\not f \phi}(\bar{\lambda}) d \mu(\lambda)=\int \not \partial \phi(-i) d \mu(\lambda) \\
& =\int \not \partial \phi(\lambda) d \check{\mu}(\dot{\lambda}), \\
& (\check{\mu}(E)=\mu(-E), \quad E=\{t ;-t \in E\}) .
\end{aligned}
$$

By the uniqueness of the spectral measure we shall obtain the following

THEOREM 3.3. In the case of real stationary distribution the spectral measure $\mu$ is symmetric with respect to 0 , viz. $\mu(E)=\mu(-E)$.

Conversely we have

THEOREM 3.4. Any distribution of the form $(3 \cdot 2)$ with symmetric measure $\mu$ is the covariance function of a certain stationary distribution which is real normal.

The proof is similar to that of Theorem $3 \cdot 2$; we use the existence theorem of real normal systems instead of that of complex normal ones.

EXAMPLE. $B^{\prime}$ is a real stationary distribution whose spectral measure is the ordinary Lebesgue measure, because we have

$$
\delta(\phi)=\phi(0)=\int \mathcal{Z} \phi(\lambda) d \lambda .
$$

\section{$\S 4$. Spectral Decomposition of Stationary Distribution}

We shall first introduce a random measure. Let $\mu$ be a nonnegative measure defined for all Borel sets in $R=(-\infty, \infty)$ and $\boldsymbol{B}^{*}$ denote the system of all Borel sets with finite $\mu$-measure. An $\mathfrak{S}$-valued function $M(E)$ defined for $E \in \boldsymbol{B}^{*}$ is called a random measure with respect to $\mu$ if and only if

$$
\left(M\left(E_{1}\right), M\left(E_{2}\right)\right)=\mu\left(E_{1} \cap E_{2}\right), E_{1}, E_{2} \in B^{*} .
$$

We get, by the definition,

$$
\begin{gathered}
\|M(E)\|^{2}=\mu(E), \\
M\left(E_{1}\right) \perp M\left(E_{2}\right) \quad \text { if } \quad E_{1} \cap E_{2}=\phi .
\end{gathered}
$$

Making use of the additivity of $\mu$ we have

$$
M(E)=\sum_{n=1}^{\infty} M\left(E_{n}\right),
$$


where $E_{1}, E_{2}, \cdots$ are disjoint to each other and belong to $B^{*}$ with their sum $E=\sum_{n=1}^{\infty} E_{n} ;\left(4 \cdot 2^{\prime \prime}\right)$ will justify the term of random measure. We can easily define the integral with respect to the measure [3] $(\mathrm{IX}, \S 2)^{4)}$ :

$$
M(f)=\int f(\lambda) d M(\lambda)
$$

for $f \in L^{2}(R, \mu) . \quad M(f)$ satisfies the following conditions :

$$
\begin{array}{cc}
(4 \cdot 4 \cdot a) \quad\left(M\left(f_{1}\right), M\left(f_{2}\right)\right)=\left(f_{1}, f_{2}\right) \quad\left(\equiv \int f_{1}(\lambda) \overline{f_{2}(\lambda)} d \mu(\lambda)\right), \\
(4 \cdot 4 \cdot b) \quad M\left(c_{1} f_{1}+c_{2} f_{2}\right)=c_{1} M\left(f_{1}\right)+c_{2} M\left(f_{2}\right) .
\end{array}
$$

Let $X(t)$ be any stationary continuous process with the spectral measure $\mu$. Then it is known [3] (XI, $\S 4)$ that $X(t)$ may be expressed in one and only one way as

$$
X(t)=\int e^{-i 2 \pi \lambda \ell} d M(\lambda)
$$

where $M$ is a random measure with respect to $\mu$. This fact was proved by many scholars, especially by A. Kolmogorov, H. Cramer [2] and M. Loève (See [9] Th. 27.2, p. 123). Therefore the stationary distribution induced by $X(t)$ may be expressed as

$$
X(\phi)=\int \mathscr{f} \phi(\lambda) d M(\lambda)=M(\not{\not} \phi) .
$$

We shall now show that this identity holds for any general stationary distribution, i.e.

THEOREM 4.1. Let $X$ be any stationary distribution with the spectral measure $\mu$. Then $X(\phi)$ will be expressible in the form (4.6) in one and one way, $M$ being a random measure with respect to $\mu$. Conversely, any random distribution of such form is a stationary distribution.

Definition. We shall call the identity $(4 \cdot 6)$ the spectral decomposition of $X$ and $M$ the spectral random measure of $X$.

PROOF OF THEOREM. We shall first remark that $\mathscr{A} D=\{\not{\not} \phi$; $\phi \in D\}$ is dense in $L^{2} \equiv L^{2}(R, \mu)$. Let \& denote the class of all rapidly decreasing functions [11] (VII, $\S 3$ ). Then $\mathscr{f}$ will be a homeomorphic mapping from \& onto itself, where the topology on $\varnothing$ is what is usually used in the theory of distributions. As $D$ is dense in $\varnothing$, so is $\mathscr{Z} D$. In making use of the condition $(3.5)$ we shall easily see that $\delta$ is included by $L^{2}$ and that ' $\varphi_{n} \rightarrow \varphi$ in

4) J. L. Doob uses a process with orthogonal increments instead of our random measure, but both concepts are equivalent. 
$\not$ ' implies ' $\varphi_{n} \rightarrow \varphi$ in $L^{2}$ '. Therefore $\not \mathscr{D}$ is dense in $\phi$ with respect to the norm of $L^{2}$. As $\varnothing \supseteq \varnothing$ and $D$ is dense in $L^{2}$, $\varnothing$ is dense in $L^{2}$ and so $\mathscr{F} D$ is dense in $L^{2}$.

The uniqueness of the expression is clear as $\mathscr{Z D}$ is dense in $L^{2}$. In order to prove the possiblity of the expression, we shall put

$$
T(\psi)=X(\phi) \text { for } \quad \psi=\not{\not} \phi .
$$

Then $T$ will be a mapping from $\mathscr{\not D}\left(\subseteq L^{2}\right)$ into $\mathfrak{S}$, which is clearly linear and isometric on account of the identity:

$$
\|T(\phi)\|^{2}=(X(\phi), X(\phi))=\rho(\phi * \widetilde{\phi})=\int\left|\psi^{\prime}(\lambda)\right|^{2} d \mu(\lambda)=\|\boldsymbol{\phi}\|^{2},
$$

since $\mathcal{Z}(\phi * \tilde{\phi})=|F \phi|^{2} . \quad \not \mathscr{D}$ being dense in $L^{2}$, we can extend $T(\psi)$ to a linear isometric mapping from $L^{2}$ into $\mathfrak{S}$. As the characteristic function $x_{E}(\lambda)$ of a set $E \in \boldsymbol{B}^{*}$ belongs to $L^{2}$, we may define $M(E)$ as follows:

$$
M(E)=T\left(x_{E}\right)
$$

Then we have

$(4 \cdot 8) \quad\left(M\left(E_{1}\right), M\left(E_{2}\right)\right)=\int x_{E_{1}}(\lambda) \overline{x_{L_{2}}(\lambda)} d \mu(\lambda)=\mu\left(E_{1} \cap E_{2}\right)$, since $T$ is isometric. In addition to this, we shall have

$$
M(f)=T(f) \text { for } f \in L^{2},
$$

for this is evidently true for any simple function $f$ in $L^{2}$ by the definition and we shall easily see that it is also true for any $f \in L^{2}$, by taking into account the fact that both sides of $(4 \cdot 10)$ are isometric in $f$ and any $f \in L^{2}$ is expressed as the $L^{2}$-limit of a sequence of simple functions. If we put $f=\not \partial \phi$ in $(4 \cdot 9)$, we obtain $(5 \cdot 6)$ at once. The last part of the theorem is clear by the definitions.

Making use of this theorem we can prove that the class of stationary processes $\mathfrak{S}^{\circ}$ coincides with $\mathfrak{S}_{0}$ in $(3 \cdot 5)$, i.e.

$$
\text { THEOREM 4.2. } \quad \varsigma^{\circ}=\mathfrak{S}_{0} \text {. }
$$

Proof. As $\mathfrak{S}^{\circ} \subseteq \mathfrak{S}_{0}$ is clear by Khintchine's decomposition $(3 \cdot 1)$, we need only prove its inverse inclusion relation. Let $X$ be any element of $\mathfrak{S}_{0}$. Then we have

$$
X(\phi)=\int \not \not \phi(i) d M(\lambda),\left(M\left(E_{1}\right), M\left(E_{2}\right)\right)=\mu\left(E_{1} \cap E_{2}\right),
$$

where

$$
\int d \mu(\lambda)<\infty
$$


Put

$$
Y(t)=\int e^{-i 2 \pi \lambda t} d M(\lambda),
$$

which may be defined, since the $\lambda$-function $e^{-i 2 \pi \lambda t}$ belongs to $L^{2}$ by virtue of $(4 \cdot 11) . \quad Y(t)$ proves to be a stationary continuous random process. Therefore we have, for $\phi \in \mathcal{D}$,

$$
\int Y(t) \phi(t) d t=\int \phi(t) \int e^{-i 2 \pi \lambda t} d M(\lambda) d t=\int \not{Z} \phi(\lambda) d M(\lambda)=X(\phi),
$$

which implies that $X(\phi)$ is induced by a process $Y \in \mathbb{S}^{\circ}$.

By the same way as in Theorem 3.3 we shall obtain

THEOREM 4.3. In case of real stationary distributions the spectral random measure $M$ is hermitian-symmetric i.e.

$$
M(E)=\overline{M(-E)} .
$$

\section{§5. Derivative of Stationary Random Distribution}

Any random distribution has derivatives of any order, which are also random distributions.

THEOREM 5.1. Let $X$ be a stationary distribution with spectral measure $\mu$ and spectral random measure $M$. Then $X^{(k)}\left(=D^{k} X\right)$ is also a stationary distribution $M$ whose spectral measure $\mu_{k}$ and spectral random measure $M_{k}$ are given by

$$
d \mu_{k}(\lambda)=(2 \pi \lambda)^{2 k} d \mu(\lambda), \quad d M_{k}(\lambda)=(i 2 \pi \lambda)^{k} d M(\lambda) .
$$

Proof. We have, by the definition,

$$
\begin{aligned}
X^{(k)}(\phi) & =(-1)^{k} X\left(\phi^{(k)}\right)=(-1)^{k} \int \mathscr{J} \phi^{(k)}(\lambda) d M(\lambda) \\
& =\int(i 2 \pi \lambda)^{k} \not{y} \phi(\lambda) d M(\lambda),
\end{aligned}
$$

since we have, for $\phi \in D$,

$$
\mathscr{f} \phi^{(k)}(\lambda)=(-1)^{k}(i 2 \pi \lambda)^{k} \mathscr{H} \phi(\lambda) .
$$

Thus $X^{(k)}$ proves to be a stationary distribution satisfying the above conditions.

By this theorem we shall see that ' $X \in S_{n}$ ' implies ' $X^{(k)} \in S_{n+k}$ '. Therefore we shall have, by Theorem $4 \cdot 2$,

THEOREM $5 \cdot 2 .{ }^{5}$ ) In order that $X^{(k)}$ is a stationary continuous process, it is necessary and sufficient that the spectral measure $\mu$ of $X$ satisfies

5) Cf. [3] XI, § 9 Example 1, p. 535. 


$$
\int \lambda^{2 k} d \mu(\lambda)<\infty \text {. }
$$

\section{§6. Continuous Process with Stationary k-th Order Increments}

Let $Y(t)$ be a continuous random process, i.e. $Y \in G(\mathfrak{g})$. The $k$-th order increment of $Y$ is defined by

$$
\Delta^{(k)} Y(t)=\Sigma_{\nu=0}^{k}\left(\begin{array}{l}
k \\
\nu
\end{array}\right)(-1)^{\nu} Y(t+\nu \Delta),
$$

where $\Delta$ is an arbitrary real number. This is also a continuous random process for any fixed $\Delta$. If $\Delta^{(k)} Y$ is a continuous random process for any fixed $\Delta$, viz.

$$
\left(\Delta^{(k)} Y(t+h), \Delta^{(k)} Y(s+h)\right)=\left(\Delta^{(k)} Y(t), \Delta^{(k)} Y(s)\right),
$$

then $Y(t)$ is called a process with stationary $k$-th order increments. The totality of such processes will be here denoted with $\Im_{k}$. The special case $k=1$ has already been discussed as a screw line in Hilbert space by J. v. Neumann and I. J. Schönberg [10] and A. Kolmogorov [8]. ${ }^{\text {) }}$ Now we shall establish a close relation between $\mathfrak{J}_{k}$ and $\mathfrak{S}_{k}$, which will give us a generalisation of the results obtained by these scholars.

THEOREM 6.1. If $Y \in \mathfrak{I}_{k}$, then $Y^{(k)} \in \mathfrak{S}_{k}$, and conversely, if $X \in \mathfrak{S}_{k}$, then there exists $Y \in \Im_{k}$ such that $X=Y^{(k)}$; Precisely speaking, if

\section{then}

$$
X(\phi)=\int \not \partial \phi(\lambda) d M(\lambda)
$$

$$
\begin{gathered}
Y(t)=\sum_{\nu=0}^{k-1} t^{\nu} A_{\nu}+\int \frac{e^{-i \Omega \pi \lambda t}-L_{k}(\lambda, t)}{(-i 2 \pi i)^{k}} d M(\lambda), \\
\left(L_{k}(\lambda, t)=\sum_{\nu=0}^{i-1}(-i 2 \pi i t)^{\nu} / \mid \nu(|\lambda| \leq 1),=0(|\lambda|>1)\right) \\
d^{(k)} Y(t)=\int e^{-i 2 \pi \lambda t}\left(\frac{1-e^{-i \imath \pi \lambda \Delta}}{-i 2 \pi \lambda}\right)^{k} d M(\lambda) .
\end{gathered}
$$

Proof. Suppose that $Y \in \mathfrak{\Im}_{k}$. Then we have

$$
\begin{aligned}
& \left(\Delta^{(k)} Y\left(\tau_{h} \phi\right), \Delta^{(k)} Y\left(\bar{\tau}_{h} \psi\right)\right) \\
= & \left.\iint\left(\Delta^{(k)} Y(t), \Delta^{(k)} Y(s)\right) \phi(t+h) \overline{\psi(s+h}\right) d t d s \\
= & \left.\iint\left(\Delta^{(k)} Y(t+h), \Delta^{(k)} Y(s+h)\right) \phi(t+h) \overline{\psi(s+h}\right) d t d s
\end{aligned}
$$

6) Cf. [3] XI, §11 and [6]. 


$$
\begin{aligned}
& =\iint\left(\Delta^{(k)} Y(t), \Delta^{(k)} Y(s)\right) \phi(t) \overline{\phi(s)} d t d s \\
& =\left(\Delta^{(k)} Y(\phi), \Delta^{(k)} Y(\phi)\right) .
\end{aligned}
$$

Hence it follows that

$$
\left(Y^{(k)}\left(\tau_{h} \phi\right), Y^{(k)}\left(\tau_{h} \phi\right)\right)=\left(Y^{(k)}(\phi), Y^{(k)}(\phi)\right)
$$

which implies $Y^{(k)} \in \mathfrak{S}$. By virture of Theorem $4 \cdot 1, Y^{(k)}$ is expressible in the following form:

(6.6) $Y^{(k)}(\phi)=\int \not{y} \phi(\lambda) d M(\lambda), \quad\left(M(E), M\left(E^{\prime}\right)\right)=\mu\left(E \cap E^{\prime}\right)$.

In order to show that $Y^{(k)} \in \mathfrak{S}_{k}$, we need only prove that

$$
\int_{|\lambda| \geq 1} \frac{d \mu(\lambda)}{\lambda^{2 k}}<\infty
$$

Put

$$
Y_{1}(\phi)=\int g_{k} \phi(\lambda) d M(\lambda)
$$

where

$$
\mathcal{G}_{k} \phi(\lambda)=\int \frac{e^{-t 2 \pi \lambda t}-L_{k}(\lambda, t)}{(-i 2 \pi \lambda)^{k}} \phi(t) d t .
$$

Since we have

$$
y_{k} \phi(\lambda)=\frac{\mathscr{y} \phi(\lambda)}{(-i 2 \pi \lambda)^{k}}, \text { for }|\lambda|>1,
$$

and $\not{\not} \phi$ is rapidly decreasing, $\mathscr{G}_{k} \phi$ belongs to $L^{2}(R, \mu)$, so that the above integral will be determined. Then we have

$$
\begin{array}{rlrl}
Y_{1}^{(k)}(\phi) & =(-1)^{k} Y_{1}\left(\phi^{(k)}\right)=(-1)^{k} \int \mathscr{G}_{k} \phi^{(k)}(i) d M(i) \\
& =\int \not \partial \phi(i) d M(i) & & \text { (by partial integration) } \\
& =Y^{(k)}(\phi) & & \text { (by }(6 \cdot 6)),
\end{array}
$$

which implies

$$
\left(Y-Y_{1}\right)^{(k)}(\phi)=0,
$$

and so $Y-Y_{1}$ may be induced by a polynomial of $t$ with coefflcients in $\mathfrak{S}$ and the degree less than $k$ :

$$
\left(Y-Y_{1}\right)(t)=\sum_{\mathrm{J}_{\nu=0}-1}^{k \nu} A_{\nu}, \quad A_{\nu} \in \mathfrak{S}, \quad 0 \leq \nu \leq k-1 ;
$$

the proof of this fact is the same as that of the corresponding fact in $D^{\prime}$ [11] (II, $\left.\S 4\right)$. Therefore we get

$$
\Delta^{(k)} Y(\phi)=\Delta^{(k)} Y_{1}(\phi)=Y_{1}\left((-\Delta)^{(k)} \phi\right)
$$


Stationary raidom distributions

$$
\begin{aligned}
& =\int \mathscr{G}_{k}(-\Delta)^{(i)} \phi(\lambda) d M(i) \\
& =\int\left(\frac{1-e^{-i 2 \pi \lambda \Delta}}{-i 2 \pi \lambda}\right)^{k} \not{f} \phi(\lambda) d M(\lambda)
\end{aligned}
$$

and so

$$
\begin{aligned}
& \left\|\Delta^{(k)} Y(\phi) \cdot\right\|^{2}=\int\left|\frac{1-e^{-i 2 \pi \lambda \Delta}}{-i 2 \pi \lambda}\right|^{2 k}|\not{d} \phi(\lambda)|^{2} d \mu(\lambda) \\
\geq & c_{1} \int_{\mid \lambda_{1} \geq 1}\left|1-e^{-i 2 \pi \lambda \Delta}\right|^{2 k}|\not{f} \phi(\lambda)|^{2} \frac{d \mu(\lambda)}{i^{2 k}} \quad\left(c_{1}=\left(\frac{1}{2 \pi}\right)^{2 k}\right) .
\end{aligned}
$$

Let $\phi_{n}(t)$ be a non-negative function $\in \mathcal{D}$ such that

$$
\phi_{n}(t)=0 \quad\left(|t| \geq \frac{1}{n}\right), \quad \int \phi_{n}(t) d t=1 .
$$

Then we have

$$
\left|\mathcal{F} \phi_{n}(\lambda)\right| \geq \frac{1}{2} \quad \text { for } \quad|\lambda| \leq \frac{n}{16},
$$

and in addition

$$
\begin{aligned}
& \left\|\Delta^{(k)} Y\left(\phi_{n}\right)\right\| \leq\left\|\int \Delta^{(k)} Y(t) \phi_{n}(t) d t\right\| \leq \int\left\|\Delta^{(k)} Y(t)\right\| \phi_{n}(t) d t \\
& \quad=\int_{-1 / n}^{1 / n}\left\|\Delta^{(k)} Y(t)\right\| \phi_{n}(t) d t \leq \max _{t(i \leq 1}\left\|\Delta^{(k)} Y(t)\right\|\left(=c_{2}(\Delta)\right)
\end{aligned}
$$

Thus we obtain, putting $\phi=\phi_{n}$ in $(6 \cdot 9)$,

$$
\begin{aligned}
& c_{2}(\Delta)^{2} \geq c_{1} \int_{: \lambda_{i} \geq 1}\left|1-e^{-i 2 \pi \lambda \Delta}\right|^{2 k}\left|\mathcal{J}^{\prime} \phi_{n}(\lambda)\right|^{2} \frac{d \mu(\lambda)}{\dot{\lambda}^{2 k}} \\
& \geq \frac{c_{1}}{4} \int_{n / i \theta \geq i \lambda: \geq 1} \mid 1-e^{-\left.i 2 \pi \lambda \Delta\right|^{2 k}} \frac{d \mu(\lambda)}{\lambda^{2 k}} .
\end{aligned}
$$

Integrating both sides in $0 \leq \Delta \leq 1$, we have

$c_{2}^{2} \geq \frac{c_{1}}{4} \int_{n l_{10} \geq ! \lambda ! \geq 1} c(\lambda) \frac{d \mu(\lambda)}{\lambda^{2 \mu}}, \quad c(\lambda)=\int_{0}^{1} \mid 1-e^{-\left.i 2 \pi \lambda \Delta\right|^{2 k}} d \Delta, c_{2}^{2}=\int_{0}^{1} c_{2}(\Delta)^{2} d \Delta$.

But it holds that

$$
c(\lambda)=\frac{1}{|2 \pi \lambda|} \int_{0}^{12 \pi \lambda \mid}\left|1-e^{-\left.i \Delta\right|^{2 k}} d \Delta \geq \frac{1}{4 \pi} \int_{0}^{2 \pi}\right| 1-e^{-\left.i \Delta\right|^{2 k}} d \Delta \quad\left(=c_{3}>0\right)
$$

for $|\lambda| \geq 1$, because if we determine a natural number $n$ so that $n \leq|\lambda| \leq n+1$, then we have

$$
c(\lambda) \geq \frac{1}{2 \pi(n+1)} \int_{0}^{2 \pi n} \mid 1-e^{-\left.i \Delta\right|^{2 k}} d \Delta
$$




$$
=\frac{n}{2 \pi(n+1)} \int_{0}^{2 \pi}\left|1-e^{-i \Delta}\right|^{2 k} d \Delta \geq \frac{1}{4 \pi} \int_{0}^{2 \pi}\left|1-e^{-i \Delta}\right|^{2 k} d \Delta .
$$

Consequently we obtain

$$
\int_{n / 1 \mathbf{B} \geq ! \lambda_{i} \geq 1} \frac{d \mu(\lambda)}{\lambda^{2 \cdot k}} \leq \frac{4 c_{2}^{2}}{c_{1} c_{3}}<\infty,
$$

which implies $(6 \cdot 7)$, as we see by letting $n$ tend to $\infty$.

Now we put

$$
Y_{2}(t)=\int \frac{e^{-12 \pi \lambda t}-L_{k}(\lambda, t)}{(-i 2 \pi \dot{\lambda})^{k}} d M(\lambda) ;
$$

this integral exists and is continuous in $t$ by virtue of $(6 \cdot 7)$. Then we have

$$
Y_{2}(\phi)=\int \phi(t) Y(t) d t=\int g_{k} \phi(\lambda) d M(\lambda)=Y_{1}(\phi) .
$$

Thus $Y_{1}(\phi)$ is defined by $Y(t)$ and so we have

$$
Y(t)=Y_{2}(t)+\sum_{\nu=0}^{k-1} t^{\nu} A_{\nu},
$$

which implies $(6 \cdot 4)$ and accordingly $(6 \cdot 5)$.

Now we shall prove the second part of the theorem. Suppose that $X(\phi) \in \mathfrak{S}_{k}$ be expressed as $(6 \cdot 3)$. Put

$$
Y_{0}(t)=\int \frac{e^{-i 2 \pi \lambda t}-L_{k}(\lambda, t)}{(-i 2 \pi \dot{\lambda})^{k}} d M(\lambda) ;
$$

as $X(\phi) \in \mathfrak{S}_{k}$, we see that this integral exists and is continuous in $t$. Then we have

$$
\begin{aligned}
Y_{0}^{(k)}(\phi) & =(-1)^{k} Y_{0}\left(\phi^{(k)}\right)=(-1)^{k} \int \mathscr{G}_{k} \phi^{(k)}(\lambda) d M(\lambda) \\
& =\int \mathcal{f} \phi(\lambda) d M(\lambda)=X(\phi) .
\end{aligned}
$$

If $Y^{(k)}(\phi)=X(\phi)$, then $\left(Y-Y_{0}\right)^{(k)}(\phi)=0$ and so

$$
Y(t)=Y_{0}(t)+\sum_{\nu=0}^{k-1} t^{\nu} A_{\nu}, \quad A_{\nu} \in \mathfrak{S} .
$$

We have further

$$
\Delta^{(k)} Y(t)=\Delta^{: k)} Y_{0}(t)=\int e^{-i 2 \pi \lambda t}\left(\frac{e^{-i 2 \pi \lambda \Delta}-1}{-i 2 \pi \lambda}\right)^{k} d M(\lambda)
$$

which implies that $\Delta^{(k)} Y(t)$ is a continuous stationary process, viz. $Y \in \mathfrak{\Im}_{k}$, since 


$$
\int\left|\frac{e^{-i 2 \pi \lambda \Delta}-1}{-i 2 \pi \lambda}\right|^{2 k} d \mu(\lambda)<\infty
$$

by virture of the assumption: $X \in \mathfrak{S}_{k}$.

By the same way as in Theorem $3 \cdot 3$ we have

THEOREM 6.2. In case of real processes $\epsilon \mathfrak{\Im}_{i}$ the above spectral random measure satisfies $M(E)=\overline{M(-E)}$ and the above coefficients $A_{\nu}, \nu=1,2, \cdots, k-1$ are real.

\section{Mathematics Department, Kyoto University.}

\section{BIBLIOGRAPHY}

1. S. Bochner: Fouriersche Integrale, Leipzig, 1932.

2. H. Cramér: On the theory of stationary random processes, Ann. Math. 41, 215-230 (1940).

3. J. L. Doob: Stochastic processes, New York, 1953.

4. K. Itô: Multiple Wiener integral, Jour. Math. Soc. Japan, 3, 1, 156-169, (1951).

5. K. Itô : Complex multiple Wiener integral, Jap. Jour. Math. 22, 63-86, (1952).

6. K. Itô: A screw line in Hilbert space and its application to probability theory, Proc. Imp. Acad. Tokyo 20, 4, 203-209, (1944).

7. A. Khintchine: Korrelations theorie des stationären stochastischen Prozesse, Math. Ann. 109, 601-615.

8. A. Kolmogorov: Kurven in Hilbertchem Raum die gegenüber eine einparametrigen Gruppe von Bewegungen invariant sind. C. R. (Doklady) Acad. Sci. U.R.S.S. 26, 6-9, (1940).

9. P. Lévy: Processus stochastiques et mouvement brownien, Paris 1948.

10. J.v. Neumann and I. J. Schönberg: Fourier integrals and metric geometry, Trans. Amer. Math. Soc. 50, 226-251 (1941).

11. L. Schwartz: Théorie des distributions 1, 2, Paris, 1950. 